\title{
Mimicking neutron star precession by polar cap current-pattern drifting
}

\author{
M. Ruderman ${ }^{1,2}$ and J. Gil ${ }^{2,3}$ \\ 1 Department of Physics and Columbia Astrophysics Laboratory, Columbia University, New York, USA \\ e-mail:mar@astro.columbia.edu \\ 2 Institute of Astronomy, Cambridge, UK \\ 3 Institute of Astronomy, University of Zielona Góra, Lubuska 2, 65-265 Zielona Góra, Poland \\ e-mail: jag@astro.ia.uz.zgora.pl
}

Received 10 August 2006 / Accepted 18 October 2006

\section{ABSTRACT}

\begin{abstract}
Aims. We propose a model for rotating current patterns within radiopulsar polar cap accelerators which has observational consequences that mimic those which have been attributed to neutron star precession.

Methods. The model is a simple extension of a commonly used one for the origin of the "drifting subpulses" often observed within the pulse envelope of radiopulsars.

Results. The new model's current pattern rotation period (with respect to the neutron star) is estimated to be of order a year. Associated with that rotation are small oscillations in spin-down torque, pulse arrival time, and radiobeam direction with this same period. These have estimated magnitudes which support a possible reinterpretation of precession "observations" which could resolve the problem of obtaining the required precession parameters with canonical neutron star models.
\end{abstract}

Key words. pulsars: general - stars: neutron

\section{Introduction}

For a considerable number of pulsars the residuals $(\Delta t)$ between observed pulse arrival times and a smooth expectation based upon average period $P, \dot{P}$, and $\ddot{P}$ can be fit to a single oscillation

$\Delta t=a \sin \left(\frac{2 \pi t}{P_{\mathrm{p}}}\right)$

and its overtones (cf. Table 1). The physical reality and significance of this residual oscillation is particularly compelling for PSR 1828-11 (Stairs et al. 2000) whose observed time variation in pulse shape is very strongly correlated with an oscillating component in $\dot{P}(t)$, with the same period $P_{\mathrm{p}}$. This correlated pulse-shape component has discouraged an explanation of the $\Delta t$ oscillation as neutron star (NS) movement in response to that of planetary companions. Instead it has generally been considered as strong evidence that this neutron star has a sustained precession with period $P_{\mathrm{p}}$ of about 3 years.

A long-lasting precession period of such magnitude is compatible with pulsar models which describe a neutron star as a rigid body (Akgün et al. 2006, this paper also contains an excellent well-referenced survey of previous work on NS precession). However, the precession parameters inferred from observations are much too long to be compatible with the canonical models of a NS core containing a neutron-superfluid and a protonTypeII-superconductor threaded by a strong magnetic field linking the core, crust, and external magnetosphere (Link 2003; see also Shaham 1977; Sedrakian et al. 1999; and a different view by Alpar 2005). In a proton-TypeII-superconductor, magnetic field is organized into quantized flux-tubes which interact
Table 1. Period $\left(P_{\mathrm{p}}\right)$ and amplitudes $(a)$ for the oscillating component in the time residuals between observations and expectations for several radiopulsars.

\begin{tabular}{cccc}
\hline \hline Pulsar & $P_{\mathrm{p}}$ (days) & $a\left(10^{-2} \mathrm{~s}\right)$ & Ref. \\
\hline Crab & $10^{3}$ & 1 & LPS88 \\
B1828-11 & $10^{3}$ & 2 & SLS00 \\
B1642-03 & $2 \times 10^{3}$ & 2 & SLU01 \\
B1929+10 & $1 \times 10^{3}$ & 1 & U03 \\
B1557-50 & $2 \times 10^{3}$ & 0.5 & CUO03 \\
RX J0720 & $3 \times 10^{3}$ & $10^{2}(?)$ & HTD06 \\
\hline
\end{tabular}

strongly with coexisting quantized vortex-lines of the spinning neutron superfluid. If, in a less standard model, the protons were to form a type I-superconductor, a "mixed state" would appear in which the magnetic field becomes strong enough in some regions to quench superconductivity there but vanishes elsewhere. According to Sedrakian (2005) the drag on vortex-lines moving inside the very different regions of such a "mixed state" can be small enough to be compatible with long-period NS precession. (However, special consideration of expected pinning or pile-up of vortices at very numerous interfaces between magnetized and unmagnetized regions still seems needed.)

The canonical model for a NS, with its strong interaction between a core's moving superfluid neutron vortex-lines and the magnetic field out through the NS's conducting crust, agrees well with a number of different kinds of pulsar observations: dipole magnetic field evolution in spinning-down pulsars, magnetic field structures of millisecond pulsars, Crab-like "glitches", giant Vela-like "glitches" (Ruderman 2005, 2006). It seems 
problematic whether a non-canonical model with a very much weaker interaction between core vortex-lines and surface magnetic should have similar consequences.

We propose below models which avoid real and potential conflicts between canonical models of neutron star interiors and the Table 1 "precession parameters". In them current-patterns in the pulsar polar caps (and magnetosphere) rotate in a polar cap $B$-field which remains fixed in a non-precessing neutron star. The estimated current-pattern rotation period $\left(P_{\mathrm{p}}\right)$ and the amplitude $(a)$ of the pulse time of arrival oscillation caused by the current rotation's associated oscillation in NS spin-down torque are both similar to those in Table 1 .

In Sect. 2 we first review an old model (Ruderman \& Sutherland 1975) for the common rapidly "drifting subpulses" observed in many radiopulsars (Backer 1973; Weltevrede et al. 2005). In it these are a consequence of $\boldsymbol{E} \times \boldsymbol{B} c / B^{2}$ drifting of patterns of "sparks" in polar cap accelerators. The estimated and observed circulation times of the rotation pattern of these sparks in the polar cap is tens of seconds. We then consider, in Sect. 3, models for a family of related "drifting spark" models which give the very different magnitudes of Table 1 .

\section{A rotating "spark" model for canonical drifting subpulses}

Deshpande \& Rankin (1999, 2001) have made the most detailed observations and analysis of narrow subpulses moving through pulse envelopes in radiopulsars. Particularly striking is their deduction of the arrangement and motion relative to the NS of very many narrow radioemission beams in a single pulsar. In their analysis of PSR B0943+10 they deduced 20 separate sources fixed on a moving "carousel wheel" rotating in the polar cap's magnetic field with a period $\sim 40 \mathrm{~s}$. Such a moving pattern has been proposed to come from a similar structure in the current flow along the open field line bundle of magnetic field lines connecting a pulsar's polar cap to its light cylinder. Electric fields are needed to maintain such outflow. Without such fields, in the corotating frame the inner magnetosphere's electric field $(\boldsymbol{E})$ and velocity $(\boldsymbol{v})$ could be everywhere zero. In the laboratory frame $\boldsymbol{v}=\boldsymbol{\Omega} \times \boldsymbol{r}, \boldsymbol{E}=\boldsymbol{v} \times \boldsymbol{B} / c$, and the inner magnetosphere would have the Goldreich-Julian charge density $\rho_{\mathrm{GJ}}=\nabla \cdot \boldsymbol{E} / 4 \pi=\boldsymbol{\Omega} \cdot \boldsymbol{B} / 2 \pi c$ at all points. Because of current flow through the light cylinder (and especially when $|\boldsymbol{\Omega} \cdot \hat{\boldsymbol{B}}|$ increases with altitude above the polar cap), more charge must continually be pulled from the polar cap to maintain that flow than can be supplied easily. The polar cap surface may not be an adequate source for the needed particles because of ion inertia, or perhaps, in some cases, because surface ions are bound at a condensed matter interface. This extreme case is sketched in Fig. 1. (If ions can be pulled out but their initial velocities are suppressed because of ion inertia the positive excess (space charge) would be inside the accelerator, near its lower outerboundary.) The model's consequences would not be qualitatively changed. If charge from the NS surface is unavailable, the $\rho=0$ gap will grow until the additional potential across it extends to a height $h$ and $e_{ \pm}$pair production can be sustained. These pairs are produced by very energetic curvature $\gamma$-rays from accelerated $\mathrm{e}^{+}$and $\mathrm{e}^{-}$in the accelerator. Copious pair production continues far above the accelerator from those $\mathrm{e}^{+}\left(\mathrm{e}^{-}\right)$which flow out from it into that region. Then because of the consequent high conductivity there, $\boldsymbol{E} \cdot \boldsymbol{B}=0$ in the plasma above the accelerator where nearly this same $V$ is maintained from the top of the accelerator to near the light cylinder at $r \sim r_{\mathrm{lc}} \equiv c / \Omega$. The indicated potentials and charges in Fig. 1

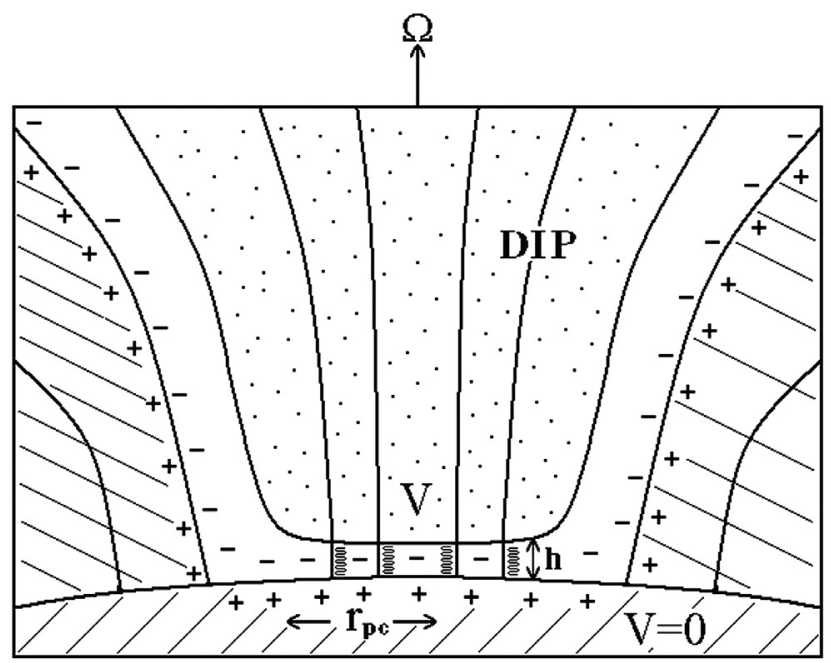

Fig. 1. Sketch of an idealized polar cap accelerator. The magnetosphere plasma on all closed magnetic field lines corotates with the neutron star (hatched regions) separated by an empty "gap" from a detached inner magnetosphere plasma (DIP). The positive charge $(+)$ which would have existed in the gap region before the accelerator formed (Goldreich $\&$ Julian charge density $\rho_{\mathrm{GJ}}$ ) is now kept from reentering the gap by the stellar surface (e.g. by ion binding to a condensed matter surface) and by the surrounding open-closed magnetic field line boundary where $\boldsymbol{E} \cdot \boldsymbol{B}=0$. (Ion inertia alone would have a qualitatively similar consequence with ion pile-up on the gap side of the surface boundary.) These form an outer surface whose potential is unchanged by the insertion of an accelerator $(V=0)$. The inner boundary of the accelerator has a constant $V\left(\sim 10^{12} \mathrm{~V}\right)$ where it is just able to sustain the $e_{ \pm}$production needed to limit accelerator growth. Effectively, the accelerator contains an additional negative charge density $\rho=-\rho_{\mathrm{GJ}}$. The inner boundary shape is just that required to make $\boldsymbol{E} \cdot \boldsymbol{B}=0$ at the inner boundary of the accelerator so there is no charged layer there and no change of $\boldsymbol{E}$ within the DIP because of the accelerator. In this model the DIP corotates with the neutron star. The minus signs (-) indicate the absence of the positive Goldreich \& Julian charge density need to keep $V=0$ everywhere.

refer only to the differences from those where $\rho=\rho_{\mathrm{GJ}}$ everywhere. The NS and all plasma on closed field lines (where $\rho$ still equals $\left.\rho_{\mathrm{GJ}}\right)$ still corotate. They are bounded by the $V=0$ surface. The upper surface of the empty accelerator zone has a fixed potential $V$ and a position and shape such that $\boldsymbol{E} \cdot \boldsymbol{B}=0$ as well as $\boldsymbol{E}$ tangential vanishing on it in the corotating frame. Then, except at the corners where the accelerator's outer boundary bends suddenly upwards along the last open field line, the accelerator thickness

$h \sim\left(\frac{V}{2 \pi \rho_{\mathrm{GJ}}}\right)^{1 / 2}$.

The entire detached inner magnetosphere plasma (DIP) above the accelerator is copiously filled with $\mathrm{e}^{ \pm}$pairs and it is assumed to have this same $V$ throughout it. It does not have any change in its initial corotation $(\boldsymbol{E} \times \boldsymbol{B}=0)$ with the NS. In this model the corotation is broken only within the accelerator where a charged particle will move with respect to the NS with an angular speed about a polar cap axis

$\Omega_{\mathrm{p}}=\left|\frac{\boldsymbol{E} \times \boldsymbol{B}}{B^{2}}\right| \frac{c}{r_{\perp}} \sim \frac{V c}{h B r_{\perp}}|\hat{\boldsymbol{E}} \times \hat{\boldsymbol{B}}|$.

$|\hat{\boldsymbol{E}} \times \hat{\boldsymbol{B}}|$, the sin of the angle between accelerator $\boldsymbol{E}$ and NS $\boldsymbol{B}$ is 0 at the model polar caps axis $\left(r_{\perp}=0\right)$ and $\sim 1$ at the corner where 
$r_{\perp} \sim r_{\mathrm{pc}}$ (radius of polar cap) and in the slot above it (see Fig. 1 for illustration).

With the very crude extrapolation $|\hat{E} \times \hat{B}| \sim r_{\perp} / r_{\mathrm{pc}}$, Eq. (3) becomes

$\Omega_{\mathrm{p}} \sim \frac{V c^{2} r_{\mathrm{pc}}}{h B_{\mathrm{d}} \Omega R^{3}}$

This estimate for $\Omega_{\mathrm{p}}$ is not sensitive to spark location ("slot", near the periphery $r_{\perp} \sim r_{\mathrm{pc}}$ or much closer to the $r_{\perp}=0$ axis), and we indicate locations of 4 exemplary sparks in Fig. 1. If the current flow pattern through the accelerator consists of particle flow in both directions bunched into "sparks" separated by a distance $\sim h$, a stable pattern of such sparks (see Beskin 1982; Gil \& Sendyk 2000) could rotate at the Eq. (4) rate (e.g. returning spark particles heat a surface spot which determines the next preferred emission spot, etc.).

For the pulsar parameters of B1828-11, $P=0.4 \mathrm{~s}$, dipole $B_{\mathrm{d}}=5 \times 10^{12} \mathrm{G}$, "characteristic spin-down age" $10^{5} \mathrm{yrs}$, polar cap radius $2 \times 10^{4} \mathrm{~cm}$ and accelerator thickness $h \sim 6 \times 10^{3} \mathrm{~cm}$ (chosen quite arbitrarily to give some partial filling of the otherwise empty accelerator gap because of ion flow into it; e.g. Gil et al. 2003) and $V \sim 10^{12} \mathrm{~V}, P_{\mathrm{p}}=2 \pi / \Omega_{\mathrm{p}} \sim 40 \mathrm{~s}$ and the number of circulating sparks on each circulating carousel wheel of the sparks pattern

$N_{\mathrm{s}} \sim \frac{2 \pi r_{\mathrm{pc}}}{h} \sim 20$.

The good agreement with $\Omega_{\mathrm{p}}$ and $N_{\mathrm{s}}$ deduced by Deshpande \& Rankin (1999) for PSR B0943+10 is comforting but far from compelling and very different kinds of models have also been proposed for such drifting subpulse observations in which outer-magnetosphere plasma plays an important role (cf. Wright 2003). Weltevrede \& colleagues (2005) have shown the lack of correlation between observed circulation periods of drifting subpulses and various other pulsar observables. Moreover, there is often great uncertainty about inferring needed polar cap accelerator parameters from those observables. (For example, in several pulsars, and probably in a large fraction of them, the polar cap magnetic field seems to be very much larger (and the polar cap area very much smaller) than the surface dipole field inferred from the pulsar's spin-down torque). A relatively robust prediction of the drifting polar cap model of Eqs. (4) and (5) comes from combining them to give a predicted angular rotation speed of the polar cap's spark-determined current pattern independent of the choice of polar cap radius or accelerator height, but depending only on the number of sparks in the carousel. For PSR B0943+10, where that number has been determined, the agreement between model and observed carousel spin-rate is good. In summary, models based upon carousels of polar cap sparks, predict carousel rotation periods (of order tens of seconds) and numbers of sparks which are not inconsistent with drifting subpulse observations.

\section{Very long period circulations of polar cap currents and their consequences}

The carousels discussed above rotate because accelerated particles from a polar cap surface make pairs, one member of which returns to the surface at a point displaced from the release point of its parent. The next generation repeats this process, but begins from the new origin. Carousel rotation periods of tens of seconds occur because the time it takes an electron (positron) from a pair created within the accelerator to return to the polar cap surface is the same as the time it spends "drifting" in the strong electric field of the accelerator. Rotation periods up to $10^{6}$ times longer than those considered above would result if the pairs were first separated far above the polar cap region (for example, in the outer-magnetosphere near the light cylinder). The time of flight back to the polar cap surface for a backflowing member of that pair would typically be huge relative the time during which it feels the strong drift fields in the relatively small polar cap accelerator.

The processes which make pairs within a polar cap accelerator continue to make them well above the accelerator. There is a large pair flux flowing up the open field line bundle from the DIP out to the light cylinder. Associated with it would be a small potential drop, $V^{\prime}$ of several $m c^{2} / e \sim 10^{6} \mathrm{~V}$, since an electric field is needed continually to adjust local net $\rho$ to $\rho_{\mathrm{GJ}}$ because of growing $|\boldsymbol{\Omega} \cdot \hat{\boldsymbol{B}}|$ along the pair flow. One way of adjusting the net charge density (generally a very small fraction of the total pair density) is by the reversal in flow direction of relatively few electrons (positrons) which then flow back toward the NS surface. The needed readjustment in charge density could well be accomplished in other ways. In the absence of enough quantitative knowledge of the open field line electric field along $B$ out to very near the light-cylinder we simply assume some backflow. If this backflow dominates that directly onto the polar cap surface from the $\mathrm{e}^{ \pm}$pairs created and separated within the accelerator, the RHS of Eqs. (4) and (5) should be decreased by about $h \Omega / c$. Then

$\Omega_{\mathrm{p}} \sim \frac{\Omega V}{B r_{\mathrm{pc}}} \sim \frac{V}{B R}\left(\frac{c \Omega}{R}\right)^{1 / 2}$.

To it should be added an additional rotation from $V^{\prime}$ with

$\Omega_{\mathrm{p}}^{\prime} \sim \frac{V^{\prime} c^{2}}{R^{3} B \Omega}$

With PSR B1828-11 parameters, $\Omega_{\mathrm{p}}^{\prime} \sim 10^{-1} \Omega_{\mathrm{p}}$ for peripheral and interior spark patterns. For slot gap sparks the gap accelerators keep about the same $\Omega_{\mathrm{p}}$ all the way up the slot so that Eqs. (4) and (5) remain valid for them. For PSR B1828-11 Eq. (6) gives $P_{\mathrm{p}} \sim 0.5 \mathrm{yr}$. For the Crab pulsar $P_{\mathrm{p}} \sim 0.1 \mathrm{yr}$.

The current distribution pattern on the polar cap and the $\boldsymbol{j} \times \boldsymbol{B}$ forces will determine the large Goldreich-Julian torque on a pulsar. We assume that associated with a polar cap current-pattern rotation should be a small oscillatory component with the same period in the pulsar's spin-down torque; $\delta \dot{\Omega}=\propto \dot{\Omega} \sin \left(\Omega_{\mathrm{p}} t\right)$. This would give an amplitude in Eq. (1) of

$a=\frac{\alpha}{2 t_{\mathrm{sd}}}\left(\frac{P_{\mathrm{p}}}{2 \pi}\right)^{2}$,

with $t_{\text {sd }}$ the characteristic spin-down age $(P / 2 \dot{P})$. We note how much larger $a$ becomes as $P_{\mathrm{p}}$ increases from the $40 \mathrm{~s}$ of Eq. (4) to $\sim 10^{7} \mathrm{~s}$ in Eq. (6).

A rough estimate of the dimensionless $\alpha$ is the product of the relative area of any expected rotating irregularity in the rotating current pattern $\left(h^{2} / r_{\mathrm{pc}}^{2}\right)$ times the fractional effect on the Goldreich-Julian spin-down torque from moving that current from one side of the polar cap to the opposite $\left(r_{\mathrm{pc}} / R\right)$, times the fraction $f(\sim 1 / 2)$ of the spin-down torque which comes from Goldreich-Julian polar cap currents. Then $\alpha \sim h^{2} / 2 r_{\mathrm{p}} R$ and, for PSR B1828-11 $a \sim 3 \times 10^{-2}$ s. For the Crab pulsar with the same $V, a \sim 0.4 \times 10^{-2}$ s. The $\Delta t$ of Eq. (1) $\propto B^{3} \Omega^{-1 / 2}$ so 
that predicted changes in $\Delta t$ are very much less than the differences of $10^{2}$ between the Crab and PSR B1828's $t_{\mathrm{sd}}{ }^{1}$. An exceptionally large amplitude would be expected and is observed for RX J0720.

\section{Summary}

Our proposed model is a very simple extension of one commonly used to describe the origin of canonical "drifting subpulses": $\boldsymbol{E} \times \boldsymbol{B}$ drift of localized $\mathrm{e}^{ \pm}$sparks within a polar-cap accelerator. It differs only in the time $\mathrm{e}^{-}\left(\mathrm{e}^{+}\right)$coming back to the stellar surface has spent inside the accelerator $\left(h / c\right.$, about $\left.10^{-7} \mathrm{~s}\right)$ relative to the time spent above it $\left(0-\Omega^{-1}\right.$, about $\left.0-10^{-1} \mathrm{~s}\right)$. If the latter time $=0$ we have the old drifting subpulse model which gives carousel circulation times of some 10's of seconds; if it is near the maximum time of flight back from near the light cylinder, we have the model of Sect. 3. (The choice between them may depend on the detailed structure of the polar cap's surface magnetic field.) The very rough estimates of Sect. 4 for $P_{\mathrm{p}}$ and $\Delta t$ are similar to those of Table 1 . While all of this is hardly a compelling argument for the kind of model proposed here it does seem to support further consideration as a not implausible way of resolving the problem of how a canonical neutron star could sustain the small precession, which has been widely suggested as the cause of very long period oscillations in timing observables of some pulsars.

Acknowledgements. We acknowledge the support of the Polish State Committee for scientific research under Grant 1 P03D 029 26. Both authors are especially grateful to the Institute of Astronomy in Cambridge for its hospitality and to the anonymous referee for very helpful suggestions.

\section{References}

Akgün, T., Link, B., \& Wasserman, I. 2006, MNRAS, 365, 635 Alpar, A. 2005, Proc. of The Electromagnetic Spectrum of Neutron Stars, ed. A. Baykal et al. (Springer)

Backer, D. C. 1973, ApJ, 182, 245

Beskin, V. 1982, SvA, 26, 4

Chukwude, A., Ubachukwu, A., \& Okeke, P. 2003, A\&A, 399, 231 (CUO03)

Deshpande, A., \& Rankin, J. 1999, ApJ, 524, 1008

Deshpande, A., \& Rankin, J. 2001, MNRAS, 322, 438

Gil, J., \& Sendyk, M. 2000, ApJ, 541, 351

Gil, J., Melikidze, G., \& Geppert, U. 2003, A\&A, 407, 315

Haberl, F., Turolla, R., de Vries, E. P., et al. 2006, A\&A, L17 (HTD06)

Link, B. 2003, Phys. Rev. Lett., 91, 101101

Lyne, A. G., Pritchard, R. S., \& Smith, F. G. 1988, MNRAS, 233, 667 (LPS88)

Ruderman, M. A., \& Sutherland, P. G. 1975, ApJ, 196, 51

Ruderman, M. A. 2005, The Electromagnetic Spectrum of Neutron Stars, ed. A. Baykal et al. (Springer), 47

Ruderman, M. A. 2006, Proc., 363, Heraeus-Seminar Neutron Stars and Pulsars, MPE Report No. 291, ed. W. Becker

Sedrakian, A., Wasserman, I., \& Cordes, J. 1999, ApJ, 524, 341

Sedrakian, A., Ph. Rev. D, 71, 3003

Shabanowa, T. V., Lyne, A. G., \& Urama, J. O. 2001, ApJ, 552, 231 (SLU01)

Shaham, J. 1977, ApJ, 214, 251

Stairs, I. H., Lyne, A. G., \& Shemar, S. L. 2000, Nature, 406, 484 (SLS00)

Urama, J. O. 2003, Proc. ASP Conf. Ser., ed. M. Bailes, D. J. Nice, \& S. E. Thorsett, 302, 107 (U03)

Weltevrede, P., Edwards, R. T., \& Stappers, B. W. 2005, A\&A, 445, 243

Wright, G. E. 2003, MNRAS, 344, 1041

\footnotetext{
1 With realistic polar cap $\boldsymbol{B} \gamma$-rays produced near a NS by a polar cap accelerator will be gravitationally bent to become a strong source of $\mathrm{e}^{ \pm}$creation in large parts of the nearby magnetosphere. Magnetic field structure can have a similar consequence. These would give $\mathrm{e}^{ \pm}$flows on open field lines which are not necessarily separated from the polar cap by the accelerator potential $V$. Indeed, such pair flow might even quench parts of that accelerator. However the argument for a $V^{\prime}$ would still hold. $\Omega_{\mathrm{p}}^{\prime}$ would still be present even in the absence of any $\Omega_{\mathrm{p}}$ for the involved $\mathrm{e}^{-} / \mathrm{e}^{+}$. Then for PSR B 1828-11 $P_{\mathrm{p}}^{\prime}=4 \mathrm{yrs}$ and for the Crab $P_{\mathrm{p}}^{\prime}=50$ yrs.
} 